Article

\title{
The DEMO Quasisymmetric Stellarator
}

\author{
Paul R. Garabedian ${ }^{1, \star}$ and Geoffrey B. McFadden ${ }^{2}$ \\ ${ }^{1}$ New York University, 251 Mercer St., New York, NY 10012, USA \\ ${ }^{2}$ National Institute of Standards and Technology, Gaithersburg, MD 20899, USA; \\ E-Mail: mcfadden@nist.gov
}

* Author to whom correspondence should be addressed; E-Mail: garabedian@ cims.nyu.edu; Tel.: +01-212-254-3046.

Received: 12 January 2010 / Accepted: 1 February 2010 / Published: 26 February 2010

\begin{abstract}
The NSTAB nonlinear stability code solves differential equations in conservation form, and the TRAN Monte Carlo test particle code tracks guiding center orbits in a fixed background, to provide simulations of equilibrium, stability, and transport in tokamaks and stellarators. These codes are well correlated with experimental observations and have been validated by convergence studies. Bifurcated 3D solutions of the $2 \mathrm{D}$ tokamak problem have been calculated that model persistent disruptions, neoclassical tearing modes (NTMs) and edge localized modes (ELMs) occurring in the International Thermonuclear Experimental Reactor (ITER), which does not pass the NSTAB simulation test for nonlinear stability. So we have designed a quasiaxially symmetric (QAS) stellarator with similar proportions as a candidate for the demonstration (DEMO) fusion reactor that does pass the test [1]. The configuration has two field periods and an exceptionally accurate $2 \mathrm{D}$ symmetry that furnishes excellent thermal confinement and good control of the prompt loss of alpha particles. Robust coils are found from a filtered form of the Biot-Savart law based on a distribution of current over a control surface for the coils and the current in the plasma defined by the equilibrium calculation. Computational science has addressed the issues of equilibrium, stability, and transport, so it remains to develop an effective plan to construct the coils and build a diverter.
\end{abstract}

Keywords: computational science; magnetic fusion; plasma physics 


\section{Computer Simulation of Magnetic Fusion}

Comparisons with both theory and experiment have shown that the NSTAB simulation of magnetic fusion in three dimensions gives reliable predictions of what can be expected to happen in practice. The variety of $3 \mathrm{D}$ solutions of the $2 \mathrm{D}$ tokamak problem that are found suggest there is little prospect a tokamak fusion reactor could become a safe and satisfactory commercial source of energy. The results of computational science show that the best resolution of these issues will be a switch to QAS or quasihelically symmetric (QHS) stellarators in the search for good candidates ( $c f$. Figure 1). Stellarators are seen to be stable and tokamaks are seen to provide satisfactory confinement, so the goal becomes to find configurations that have both these properties simultaneously.

Figure 1. Magnetic fusion of hydrogen forms helium and emits energetic neutrons from a torus of plasma shaped to optimize confinement. Modular coils generate a field keeping the ions from hitting walls.

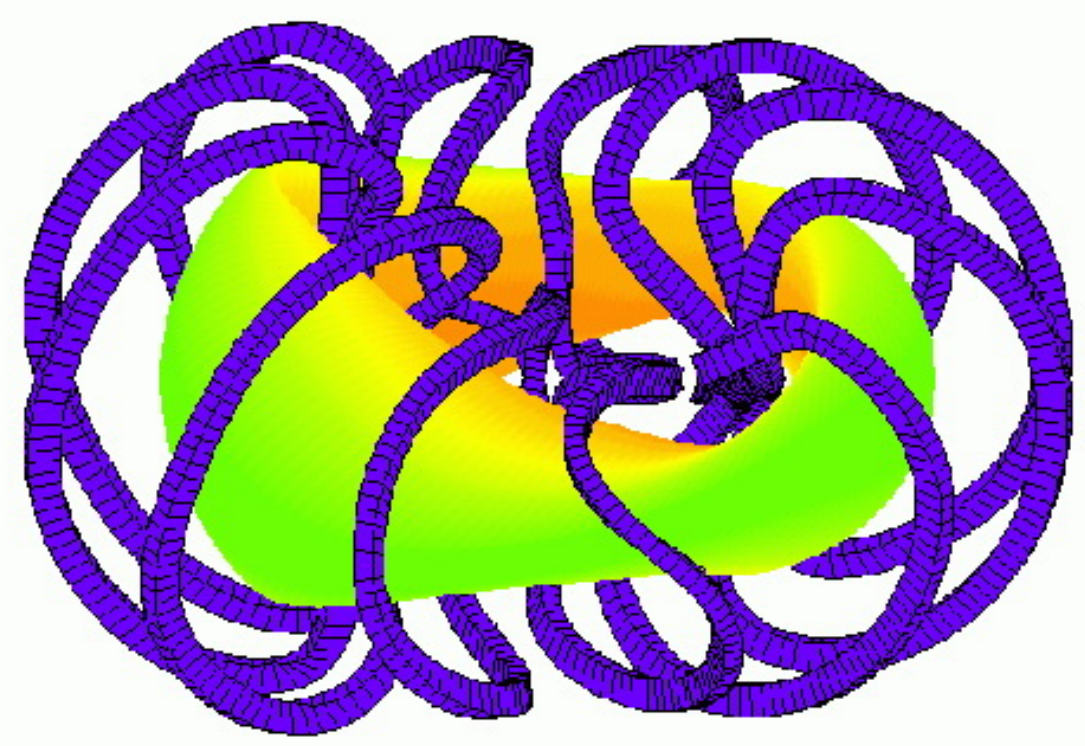

It is fortunate and remarkable that MHD equilibria with both of these features have been discovered, and it is important that this success should not get lost in the emphasis on magnetic fusion funding of the less promising ITER project.

Figure 2 displays coils of the QAS stellarator designed for a reactor showing that they are well separated and only moderately twisted. This configuration is MHD stable for realistic changes in the shape of the plasma. The NSTAB simulation of magnetic fusion predicts poor performance of ITER, but it shows that a QAS stellarator with comparable specifications would be a good reactor. An experimental verification that the required superconducting coils and magnets can be constructed and supported remains an open question in nuclear engineering. 
Figure 2. Three distinct coils of the QAS stellarator with just two field periods. When the torus of plasma is removed the coils are seen to be well enough separated so they can be constructed on the length scale of a reactor.

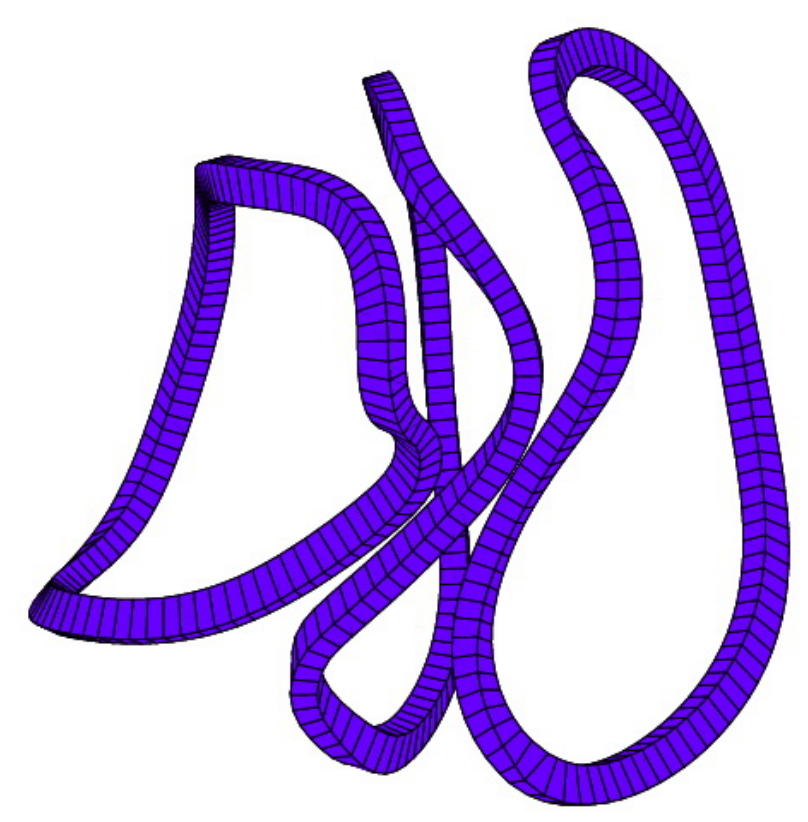

The discovery of QAS and QHS configurations has brought us to a turning point in magnetic fusion research that makes successful solutions of the problems with equilibrium, stability, and transport inside the plasma tractable. However, a review of the LHD project in Japan and the W7-X project in Germany indicates that the cost of an experiment to find out if stellarators can achieve the temperatures near $10 \mathrm{keV}$ required for fusion of hydrogen isotopes may be as high as several billion dollars, bringing it close to the specifications for ITER. It would be desirable to start with a less ambitious proposal with more modest goals. A useful project would be to study questions of mechanical and nuclear engineering that arise in the construction of magnets and modular coils.

\section{Equilibria with Stellarator Stability and Tokamak Confinement}

In Figure 3 we display a bifurcated 3D tokamak equilibrium that explains why the NSTAB simulation of tokamaks like ITER predicts that they will exhibit disruptions and crashes that make them poor candidates for a fusion reactor. The solution converges to the level of roundoff error, showing that the discrete equations can be solved exactly ( $c f$. Figure 4). A transition from stability to instability calculated as the amplitude of a resonant perturbation increases is consistent with the intermittent disruptions and crashes that are observed in tokamak experiments. Therefore we propose to switch to a QAS stellarator with enough external magnetic field to provide stability, combined with $2 \mathrm{D}$ quasisymmetry adequate to assure good confinement.

Both theory and experiment show that well designed stellarators are surprisingly MHD stable up to the energy $\beta=0.04$ required of a reactor, but their confinement is inadequate without $2 \mathrm{D}$ symmetry because trapped particles move from one drift surface to another and escape rapidly. On the other hand, 
axially symmetric tokamaks have good confinement, but they are subject to intermittent disruptions and crashes because of MHD instabilities driven by the net toroidal current that provides a poloidal field. At present the only way known to achieve both stability and good confinement simultaneously is to switch to 3D configurations with either QAS or QHS magnetic spectra. In this paper we describe such an approach based on a compact stellarator with aspect ratio $A=2.5$ like ITER that has exceptionally accurate $2 \mathrm{D}$ symmetry.

Figure 3. Cross section of an unstable tokamak equilibrium displaying a magnetic island at $\iota=0.667$. With $\beta=0.035$ and toroidal flux in the interval $0<s<1$ we put pressure $p=0.037\left(1-s^{1.8}\right)^{1.8}$ and rotational transform $\iota=0.880-0.322 s$. The $2 \mathrm{D}$ solution is stable for small perturbations of the plasma, but becomes unstable for larger 3D displacements. This behavior is symptomatic of NTMs and ELMs.

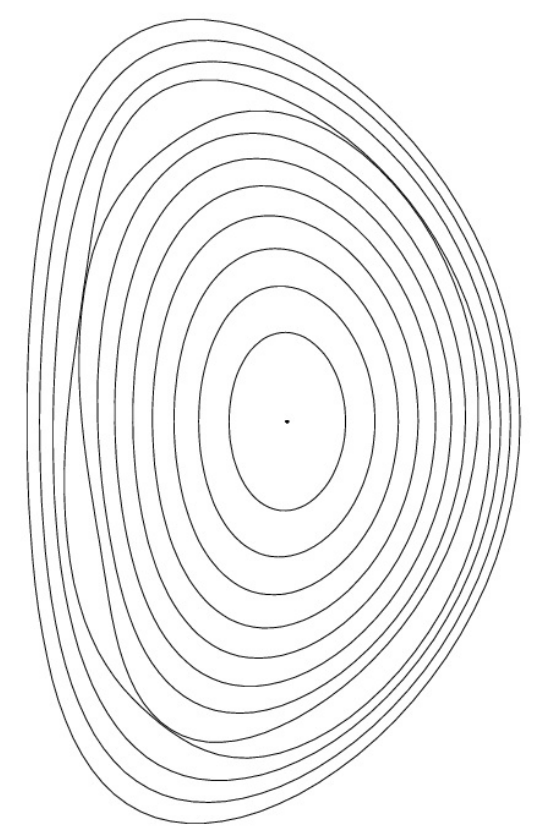

Optimized QAS and QHS stellarators are designed by careful selection of the shape of the torus of plasma confined by the magnetic field. In practice this is accomplished by running the equilibrium NSTAB code to find an appropriate solution of the MHD equations. Afterwards the TRAN Monte Carlo code is applied to a test particle model of the guiding center orbits of the hydrogen ions to evaluate transport. To validate the results of the computations the theoretical confinement time of the particles is compared with three times the energy confinement time observed in experiments. Satisfactory agreement is obtained when quasineutrality is imposed on the choice of the electric potential.

There is an extensive and complete literature about weak solutions of the nonlinear equations for MHD equilibria that can be found from the reference cited at the end of this paper [1]. A detailed presentation of the material exceeds the limitations of the review we are able to give here and required years of research. Essential information is provided by a listing of the NSTAB computer code that we are 
now making available. Convergence proofs and numerical examples are included in articles and books on the subject. Further study of such matters would also be desirable.

Figure 4. Convergence of the discrete 3D solution of a 2D tokamak problem to the level of roundoff error after $10^{7}$ iteration cycles. This NSTAB simulation confirms the risk of disruptions and ELMs crashes in the ITER project.
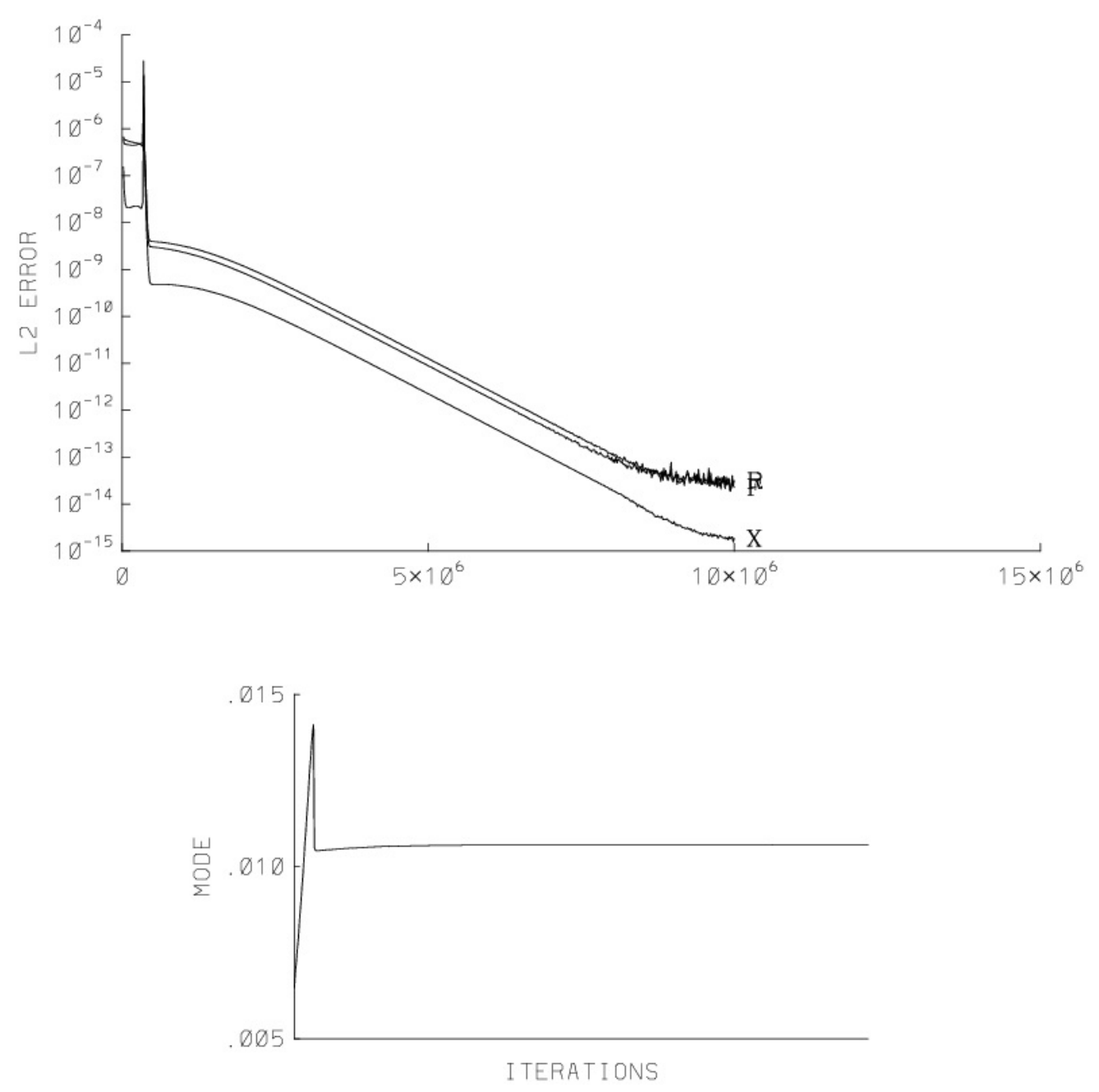

Because many tokamak experiments have had disruptions it is not justifiable to suppose that ITER can lead the way to a safely stable magnetic fusion reactor. But it is also not easy to think of an economical smaller experiment to check out the prospects for a QAS reactor. Problems with the size of the coils are tractable on the large scale of a reactor, but they become difficult on the more limited scale of a modest experiment to confirm good performance of the concept at moderate expense. New ideas to deal with these issues are needed.

\section{Computational Science of Equilibrium, Stability, and Transport}

The partial differential equations of MHD equilibrium in plasma physics are identical with the Euler equations for steady flow of an incompressible, inviscid fluid. The aerospace industry designs modern 
aircraft using a conservation form of the equations that captures shock waves accurately. A comparable method is required in plasma physics to approximate force balance correctly. Such a procedure is implemented in the NSTAB code, which explains its success in simulating magnetic fusion.

The usual way to solve the Euler equations numerically is to run the time-dependent case, subject to an appropriate radiation condition at infinity, until a steady state is reached. This fails in the application to MHD equilibrium because the plasma is confined to a torus that is bounded. We therefore replace the time-dependent scheme by a mathematical method of accelerated steepest descent that is suggested by the MHD variational principle. The ability of the LHD experiment in Japan to achieve values of $\beta$ above 0.04 helps to justify this approach to the problem in its role as a simulation of magnetic fusion with toroidal geometry.

The use of the variational principle in the NSTAB equilibrium calculation provides an effective criterion to determine stability. Uniqueness of the solution implies stability, whereas the occurrence of a second, or bifurcated, equilibrium suggests instability. The concept is implemented by introducing a dangerous mode in the iterative scheme temporarily and then examining what happens after it is removed. This diagnostic is especially decisive in the case of tokamaks, where the prevalence of 3D equilibria explains why disruptions and ELMs crashes are expected to occur at least intermittently in tokamaks. On the other hand, the external magnetic field avoids the need for net current and serves to stabilize stellarators that have an adequate magnetic well. These features of the computational theory are consistent with observations in major experiments.

In Figure 5 we exhibit four cross sections of the flux surfaces of an optimized QAS stellarator with two field periods showing how an NSTAB computation serves to establish nonlinear stability. After an interval of the toroidal flux $s$ in which there is reversed shear providing good magnetic surfaces, the rotational transform falls at the edge of the plasma to a value near 0.5 that produces four slender magnetic islands suitable for a helical diverter. The configuration is linearly unstable, but convergence of the bifurcated equilibrium in the figure to the level of roundoff error confirms its nonlinear stability to low order MHD modes.

The NSTAB and TRAN computer codes provide realistic calculations of a conservation form of the mathematical equations employed to design magnetic fusion reactors. It is essential in this context to use $3 \mathrm{D}$ rather than 2D models of the physical problem. The 2D methods that have been applied in the ITER project are not adequate and have produced misleading results. We recommend that $3 \mathrm{D}$ computations be introduced to establish more reliable predictions about the performance of the experiment and model the physics more accurately. In particular, nonlinear theory shows that large perturbations of a tokamak often destroy the plasma through a major disruption.

The numerical solution of equilibrium equations in conservation form and the estimation of test particle orbits implemented in the NSTAB and TRAN computer codes comprise a plausible description of plasma dynamics on the time scale of a reactor. The same is not true, however, of calculations based on the time-dependent version of the MHD equations, which apply to more rapid motion. Thus the 2D models widely accepted by the fusion community lead to oversimplifications and misconceptions that we avoid in the NSTAB simulation of magnetic fusion. 
Figure 5. Flux surfaces at four cross sections over the two field periods of a QAS stellarator equilibrium at $\beta=0.04$. For toroidal flux in the interval $0<s<1$ we put pressure $p=0.039\left(1-s^{2}\right)^{1.5}$ and rotational transform $\iota=0.55-0.40(0.68-s)^{2}$. This configuration is a good candidate for DEMO because its geometry is similar to that of the ITER tokamak. There is enough flexibility in the shaping of the plasma to control the stellarator contribution to $\iota$ so as to compensate for unforeseen complications with the bootstrap current.
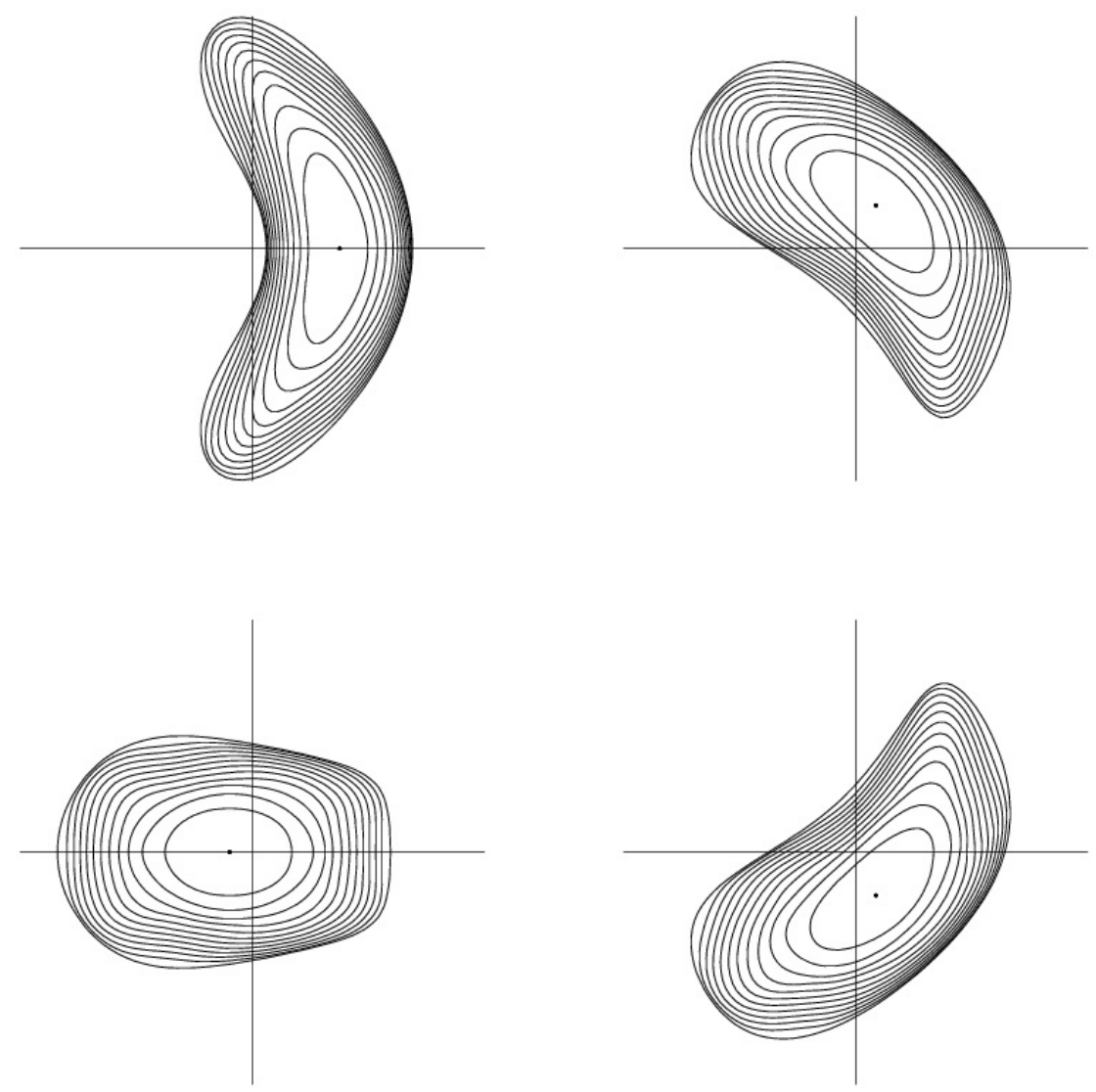

The NSTAB code imposes a nested surface hypothesis on the torus of plasma inside a system of modular coils. It becomes possible to prescribe both the pressure profile and the profile of rotational transform when conservation form of the MHD equations is employed to calculate weak solutions of the equations because they capture magnetic islands as discontinuities like current sheets at singularities in the KAM theory of dynamical systems. The physics of the plasma inside the torus is accurately described in this way, and the islands appear as pairs of flux surfaces that touch each other. Advances in numerical linear algebra and matrix iterative analysis have made this more complicated approach to the variational principle tractable in applications to nuclear fusion.

\section{The DEMO Fusion Reactor}

Numerical solution of the MHD equations in conservation form, combined with tracking orbits of test particles, make runs of the NSTAB and TRAN computer codes a reliable simulation of the performance 
of magnetic fusion reactors. They take advantage of the more refined mathematical methods in $3 \mathrm{D}$ that we have reviewed. In particular, we reject the belief that quasineutrality is intrinsic in $2 \mathrm{D}$ configurations because of conservation of angular momentum, which ignores difficulties with mean free paths measured in kilometers and with neglect of the displacement current. The success of the LHD experiment in achieving a value of $\beta$ as high as 0.04 provides evidence that our conclusions are physically realistic, for they agree with the observations.

We are concerned with the search for a QHS or QAS candidate for the DEMO magnetic fusion reactor. There is a QHS relative of the W7-X experiment that is on the list and has no net current because the rotational transform has been increased after eliminating a requirement of no bootstrap current. Unfortunately the $\mathrm{W} 7-\mathrm{X}$ itself has a dangerous resonance at the boundary of the plasma, where the rotational transform $\iota=1$, which may interfere with good performance. Our preferred candidate is a QAS stellarator with two field periods and a low aspect ratio $A=2.5$ close to that of ITER. This example has exceptional 2D symmetry, so both stability and confinement become acceptable. Detailed documentation and specifications have been published elsewhere [1].

\section{References}

1. Garabedian, P.R.; McFadden, G.B. Design of the DEMO fusion reactor following ITER. J. Res. NIST. 2009, 114, 229-236. Available online: http://www.nist.gov/jres (accessed on 20 February 2010).

(c) 2010 by the authors; licensee Molecular Diversity Preservation International, Basel, Switzerland. This article is an open-access article distributed under the terms and conditions of the Creative Commons Attribution license http://creativecommons.org/licenses/by/3.0/. 